Research Report

\title{
Age differences of multivariate network expressions during task-switching and their associations with behavior
}

\author{
Yunglin Gazes, Brian C. Rakitin, Christian Habeck, Jason Steffener, Yaakov Stern* \\ Columbia University, Taub Institute, 630 West 168th Street, P E S Box 16, New York, NY 10032, United States
}

\section{A R T I C L E I N F O}

\section{Article history:}

Received 12 March 2012

Received in revised form

14 September 2012

Accepted 20 September 2012

Available online 27 September 2012

Keywords:

Brain behavior correlation

FMRI

Multivariate analysis

Executive control

Aging

Dual-task

\begin{abstract}
A B S T R A C T
The effect of aging on functional network activation associated with task-switching was examined in 24 young (age $=25.2 \pm 2.73$ years) and 23 older adults (age $=65.2 \pm 2.65$ years) using functional Magnetic Resonance Imaging (fMRI). The study goals were to (1) identify a network shared by both young and older adults, (2) identify additional networks in each age group, and (3) examine the relationship between the networks identified and behavioral performance in task-switching. Ordinal Trend Covariance Analysis was used to identify the networks, which takes advantage of increasing activation with greater task demand to isolate the network of regions recruited by task-switching. Two taskrelated networks were found: a shared network that was strongly expressed by both young and older adults and a second network identified in the young data that was residualized from the shared network. Both networks consisted of regions associated with task-switching in previous studies including the middle frontal gyrus, the precentral gyrus, the anterior cingulate, and the superior parietal lobule. Not only was pattern expression of the shared network associated with reaction time in both age groups, the difference in the pattern expression across task conditions (task-switch minus single-task) was also correlated with the difference in RT across task conditions. On the contrary, expression of the young-residual network showed a large age effect such that older adults do not increase expression of the network with greater task demand as young adults do and correlation between expression and accuracy was significant only for young adults. Thus, while a network related to RT is preserved in older adults, a different network related to accuracy is disrupted.
\end{abstract}

(c) 2012 Elsevier Ltd. All rights reserved.

\section{Introduction}

Aging is associated with decline in behavioral performance (Salthouse, 1996; Verhaeghen \& Cerella, 2002) as well as altered neural activation (Eyler, Sherzai, Kaup, \& Jeste, 2011; Rajah \& D'Esposito, 2005), but how behavior relates to changes in neural activation in studies of aging is still unclear despite the vast number of investigations published (Eyler, et al., 2011). Understanding how aging effects on behavior relate to changes in neural activation may play a crucial role in devising preventive as well as corrective interventions for age-related decline (Lustig, Shah, Seidler, \& Reuter-Lorenz, 2009). Given that a major cognitive change associated with aging is cognitive control (Drag \& Bieliauskas, 2009), we selected a task designed by Koechlin, Ody, and Kouneiher (2003) that manipulates levels of cognitive control and has been shown to activate increasingly rostral regions of the lateral prefrontal cortex. Of the manipulations in Koechlin et al.'s task, we chose the contextual manipulation

\footnotetext{
* Corresponding author. Tel.: +1 212342 1350; fax: +1 2123421838

E-mail address: ys11@columbia.edu (Y. Stern).
}

because it was the highest manipulation level that elderly participants were able to perform based on the results from a previous behavioral study (Rakitin \& Stern, in preparation). The current study uses functional Magnetic Resonance Imaging (fMRI) to investigate the relationship between activation of brain regions and task performance in both young and elderly adults.

According to Koechlin et al. (2003), the contextual manipulation examines neuronal changes in response to selecting the appropriate stimulus-response associations cued by the contextual signal, which is the color of the stimuli. One color cued for upper/lower-case discrimination while a second color cued for vowel/consonant discrimination. Thus, the two contextual conditions consisted of single-task, in which only one color cue was used to signal one of the stimulus-response pairs, and of dualtask, in which two color cues were used to signal the two different stimulus-response pairs, placing task-switching demands on the participant. Task-switching involves switching between two or more tasks, which hinders task execution relative to performing each task alone (Rogers \& Monsell, 1995). The performance decrement due to switching between tasks is categorized into local and global switch costs. Local switch cost compares reaction time (RT) for switch trials (trials in which the task is changed 
from the previous trial) with RT in repeat trials (trials in which the task is the same as in the previous trial) while global switch cost compares RT in single-task blocks with non-switch RT in blocks containing both tasks (task-switch blocks). While global switch cost may be due to the maintenance of several task sets (sets of stimulus-response mappings) in working memory (Kray \& Lindenberger, 2000), source of the local switch cost may be attributed to various factors such as the delay in reconfiguring the task-set when a switch occurs (Rogers \& Monsell, 1995), overriding the inertia from activation of the previous task-set (Allport, Styles, \& Hsieh, 1994), as well as overcoming the residual inhibition of the current task-set due to previous performance of a competing task (Mayr \& Keele, 2000).

$>$ In the task-switching literature, both the stimuli and the responses in the task are categorized as bivalent because they are interchangeable for both tasks. Bivalent stimulus and response induce greater switch cost than univalent stimulus and response, likely due to the greater effort in activating the relevant task-set (Crone, Wendelken, Donohue, \& Bunge, 2006; Meiran, 2000). The color cues are intrinsic properties of the stimuli, rather than external, in which the task cue is a separate stimulus from the target stimulus. Most aging studies of task-switching have used bivalent stimuli but with cues that are external to the stimuli and usually appearing before stimulus presentation (Karayanidis, Whitson, Heathcote, \& Michie, 2011; Kramer, Hahn, \& Gopher, 1999). Findings on the age-related changes in task-switching behavioral performance are more consistent than on aging effects of fMRI activation associated with task-switching. In older adults, global switch cost has generally been found to be greater than local switch cost (Kray \& Lindenberger, 2000; Mayr, 2001; Verhaeghen \& Cerella, 2002). fMRI studies have found age differences in the activation associated with task-switching in distributed areas such as the prefrontal cortices, the parietal lobes, and striatal regions (Gold, Powell, Xuan, Jicha, \& Smith, 2010; Jimura \& Braver, 2010; Madden et al., 2010). Two recent functional imaging studies tried to distinguish the neuronal effects of local and global switch cost using a mixed design that combines the block and the event-related design in the same model, with the local switch cost modeled by event-related predictors and global switch cost modeled by the block predictors. Jimura and Braver (2010) reported lower sustained activity, a measure of global switch cost, and increased transient activity, a measure of local switch cost, in anterior PFC while Madden et al. (2010) observed reduced sustained activation in left inferior frontal gyrus but no age difference in transient activity in any of the task-related regions even though both studies used the same behavioral task. Jimura and Braver (2010) further investigated the relationship between brain activity and switch cost. In young adults, sustained activity in the anterior PFC correlated negatively with switch cost, but in older adults correlation with behavior was not found in sustained activity. Rather, older adults exhibited positive correlation between transient activity in the anterior PFC and switch cost, a relationship opposite that in the young adults.

While most aging studies examine age-related differences, brain activations that are unchanged by aging are also essential to understanding the aging brain. Our study had three goals: (1) to identify a common task-related network shared by young and older adults, (2) to identify any age-unique networks in addition to the shared network, and (3) to examine any agerelated difference in the relationship between brain activation and behavior in order to examine the source of performance change in task-switching. A difficulty in detecting changes in brain activation associated with increasing task difficulty is that the task-related changes are usually masked by the high intersubject variability. Therefore, Ordinal Trend Covariance analysis (OrT), a guided multivariate technique specifically developed to increase sensitivity to task-related changes, was used. It is an established multivariate technique that identifies a set of regions that consistently show covarying changes in activation with increasing task difficulty across subjects (Habeck et al., 2005). The underlying assumption is that regions involved in task processing increases in activation with greater task difficulty. Age differences could be found if a significant OrT pattern could not be identified or if the expression scores, a measure of the degree participants express a network, were significantly different between the two age groups.

\section{Method}

\subsection{Participants}

Twenty-four young and 23 older participants participated in this study (see Table 1 for more information). Participants were recruited using established market mailing procedures to equalize the recruitment procedures of young and old. Participants who responded to the mailing were telephone screened to ensure that they met basic inclusion criteria (right handed, English speaking, no psychiatric or neurological disorders, normal or corrected-to-normal vision, etc.) Individuals that passed the telephone screen were further screened in person and a Mattis Dementia Rating Scale score of at least 133 was required for retention in the study. Informed consent, as approved by the Internal Review Board of the College of Physicians and Surgeons of Columbia University, was obtained prior to study participation, and after the nature and risks of the study were explained. Participants were paid for their participation in the study.

\subsection{Activation task}

The task was based on Experiment 2 in Koechlin et al. (2003), an intrinsically cued task-switching paradigm with a no-go component, in which the color of each stimulus served as the task cue. Subjects were presented a series of four conditions comprised of two single-task conditions (Fig. 1B) and two identical task-switching conditions (Fig. 1C), with the duplication serving to match the number of trials for each discrimination (see below) between the single and taskswitching conditions. Each condition was implemented in blocks lasting $33.6 \mathrm{~s}$, comprised of 12 sequential letters (or trials). Fig. 1A shows the temporal structure of the task. Each stimulus was terminated when a response was made before deadline or after $1900 \mathrm{~ms}$, whichever came first. Stimuli onsets were separated by $2400 \mathrm{~ms}$. These trial dynamics were selected based on performance characteristics of the older adults in behavioral pilot studies, and deviate from Koechlin's briefer presentations (Koechlin et al., 2003). Each block was preceded by an instruction cue, shown for $4.8 \mathrm{~s}$, to inform the subject of the appropriate action for each stimulus. Subjects responded to each letter with a right-hand/left-hand button press or by making no action at all. For example, in the single-task condition (left of Fig. 1B), a green letter was associated with the vowel/consonant task (left press for vowel, right press for consonant) and a white letter was associated with no action, whereas in the task-switching conditions (Fig. 1C), a green letter is still associated with the vowel/consonant task and a white letter still means no action, the appearance of a red letter signals lower/upper-case identification (left press for lower-case, right press for upper-case).

In addition to the four active conditions, there were two resting conditions $33.6 \mathrm{~s}$ in duration; no stimuli were presented and no response was required. The two resting conditions were identical, but were enumerated separately to simplify the description of the Latin Square design (see below). Each resting block presented an instruction cue ("REST") followed by a blank screen. During fMRI acquisition, each subject was given six repetitions of each of the four active and two resting conditions, for a total of 36 blocks. Conditions were presented in a $6 \times 6$ fully balanced Latin Square design. The fMRI data acquisition protocol requires stopping the scanner after every six blocks, typically requiring less than $30 \mathrm{~s}$, resulting in the total session duration of approximately $26 \mathrm{~min}$ and a total of six fMRI runs with six blocks in each run.

In order to promote the scanning of participants in a stable behavioral and cognitive state, participants were pre-trained on the task and then tested on the

Table 1

Participant information.

\begin{tabular}{lllllll}
\hline & $n$ & Age (years) & Sex & Education (years) & DRS & \multicolumn{1}{l}{ NART } \\
\hline Young & 24 & $25.2 \pm 2.73$ & $13 \mathrm{~F}$ & $15.6 \pm 2.22$ & $141 \pm 2.41$ & $96.8 \pm 8.15$ \\
Old & 23 & $65.2 \pm 2.65$ & $12 \mathrm{~F}$ & $15.1 \pm 3.07$ & $140 \pm 3.14$ & $102 \pm 9.68$ \\
\hline
\end{tabular}

Note: DRS total=Mattis Dementia Rating Scale and NART $I Q=$ North American Reading Test. 
A

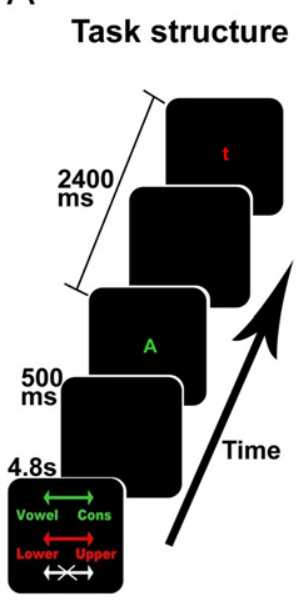

B

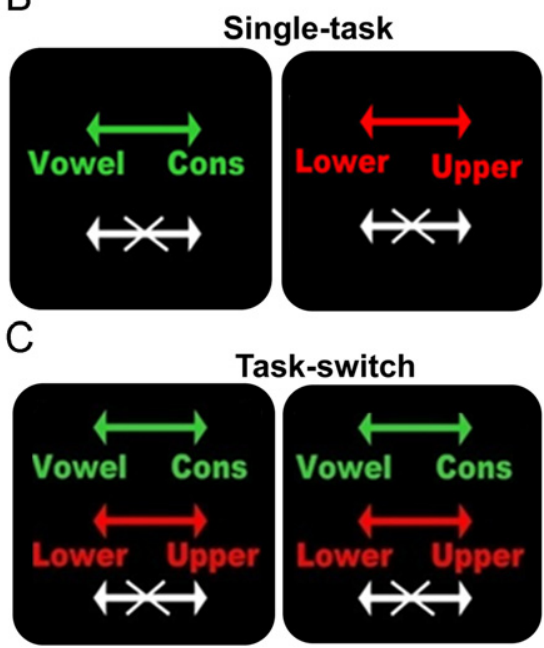

Fig. 1. (A) An example of the beginning of a block showing the instruction screen for a task-switching block, intertrial interval, a stimulus for the vowel/consonant task, intertrial interval, and a stimulus for the upper-/lower-case task. (B) and (C) show the instruction screens for the single-task conditions (B) and for the taskswitching conditions. The colors served as task-cues: green for the vowel/ consonant task, red for the upper-/lower-case task, and white for no-go trials Arrows show the response-hand assignments: left for vowel/right for consonant and left for lower-case/right for upper-case.

entire paradigm in a quiet office prior to the MRI scanning session. Training consisted of giving between one and three blocks of each condition, with unlimited time to inspect the instructions and instruction cues preceding each block, and with auditory feedback indicating incorrect responses. Then participants were tested on the entire $6 \times 6$ Latin Square identical to the testing protocol described above (pre-scan phase).

\subsection{Stimulus presentation}

Task stimuli were back-projected onto a screen located at the foot of the MRI bed using an LCD projector. Participants viewed the screen via a mirror system located in the head coil and, if needed, had vision corrected to normal using MR compatible glasses (manufactured by SafeVision, LLC. Webster Groves, MO). Responses were made on a LUMItouch response system (Photon Control Company) using the index fingers. Task administration and collection of RT and accuracy data were controlled using PsyScope 5X B53 (Macwhinney, Cohen, \& Provost, 1997) running on a Macintosh G3/G4 iBook. Task onset was electronically synchronized with the MRI acquisition computer. A MellonIOLabs Systems USB Button Box provided digital input-output for the response system and synchronization with the MRI acquisition computer, as well as millisecond accurate timing of responses.

\subsection{Image acquisition procedure}

MRI images were acquired in a 3.0 T Philips Achieva Magnet using a standard quadrature head coil. A T1-weighted scout image was acquired to determine subject position. One hundred and sixty five contiguous $1 \mathrm{~mm}$ coronal T1-weighted images of the whole brain were acquired for each subject with an MPRAGE sequence using the following parameters: TR $6.5 \mathrm{~ms}$, TE $3 \mathrm{~ms}$; flip angle $8^{\circ}$, acquisition matrix $256 \times 256$ and $240 \mathrm{~mm}$ field of view. Six functional scan sets were acquired, each of which included collection of 111 functional images acquired using a field echo echo-planar imaging (FE-EPI) sequence TE/TR $=20 \mathrm{~ms} /$ $2000 \mathrm{~ms}$; flip angle $=72^{\circ} ; 112 \times 112$ matrix; in-plane voxel size $=2.0 \mathrm{~mm} \times$ $2.0 \mathrm{~mm}$; slice thickness $=3.0 \mathrm{~mm}$ (no gap); 41 transverse slices per volume. Before the initiation of the executive task, four volumes were acquired and discarded to allow transverse magnetization immediately after radio-frequency excitation to approach its steady-state value. Any T1 scans with potentially clinically significant findings, such as abnormal neural structure, were reviewed by a neuroradiologist and removed from the sample prior to the current analysis. However, no clinically significant findings were identified or removed.

\subsection{Behavioral analysis}

Accuracy and reaction time (RT) were assessed in repeated-measures analysis of variance (ANOVA) with Group (Young/Old) as the between-subject factor, and Condition (Single/Task-switch) and Phase (pre-scan/in-scan) as the within-subject

factors. Analysis for accuracy had the additional factor of Task (Go/No-go) as a within-subject factor. This factor was not available for RT analysis because RTs could only be recorded for Go trials. Analyses were performed using Statistical Analysis Systems (SAS), v. 9.2.

\subsection{Functional MRI data pre-processing}

All image pre-processing and statistical analyses were implemented using the SPM5 program (Wellcome Department of Cognitive Neurology) (Friston, 2007) and other in-house code written in MatLab 7.10 (Mathworks, Natick MA). Images were visually inspected for imaging artifacts and neurological pathology and manually aligned along the AC-PC line. For each of the six functional datasets (corresponding to the six block types in the experimental design) from each participant, images were temporally shifted to correct for slice acquisition order using the first slice acquired in the TR as the reference and were corrected for motion by realigning to the first volume of the first session. The T1-weighted (structural) image was co-registered to the first functional volume using mutual information co-registration algorithm implemented in SPM5. This co-registered high-resolution image was then used to determine the $7 \times 8 \times 7$ non-linear basis function parameters for transformation into a standard space defined by the Montreal Neurologic Institute (MNI) template brain supplied with SPM5. This transformation was then applied to the functional data, which were re-sliced using sinc-interpolation to $2 \times 2 \times 2 \mathrm{~mm}$ and finally spatially smoothed with an $8 \mathrm{~mm}$ FWHM kernel. Both the data and the time-series design matrix were highpass filtered with a filter cutoff period of $128 \mathrm{~s}$. Autocorrelations within the timeseries were corrected for by pre-whitening the data.

\section{7. $f M R I$ subject-level processing}

For each participant's data, a block-based analysis was performed such that the predictor variables in the first-level design matrix were composed of epochs representing each unique experimental task block; each of six runs was separately modeled within which one predictor for each of four task blocks and one predictor for instructions were modeled. (The rest blocks were implicitly modeled.) Each epoch was convolved with a model of the hemodynamic response function supplied with SPM5. Contrasts for single-task and task-switch conditions were entered into the group-level analysis.

\subsection{Age-common covariance patterns}

Two steps were performed on the first-level individual data: Ordinal Trend Covariance Analysis (OrT) was performed on all of the data, then OrT was performed a second time on the residualized data for each group. A detailed explanation of each step is presented below.

\subsubsection{Group-level covariance analysis}

OrT was performed on the two task conditions to identify a task-related pattern. This analysis is similar to other regional covariance analyses techniques, notably partial least squares, to the extent that it applies principal components analysis (PCA) to the data matrix that is transformed using a matrix representing the experimental design (McIntosh, Bookstein, Haxby, \& Grady, 1996; Worsley, Poline, Friston, \& Evans, 1997). The design matrix of OrT has been optimized to increase the variance contribution (and subsequent detectability in the PCA) of effects that show consistency in their behavior across conditions and subjects (Habeck et al., 2005). A subsequent linear regression is employed to identify a covariance pattern in the MR signal with increasing mean subject expression across the single-task and the task-switch conditions based on a linear combination involving a small set of principal components. The property of a consistent monotonic within-subject change of pattern expression across task conditions (beyond mere mean change) is called an "ordinal trend". The combination of a PCA after data transformation with our specialized design matrix and a subsequent linear regression is very sensitive to the presence of ordinal trends in the data (Habeck, et al., 2005) and will increase the chance of finding a pattern that shows an increase in expression across the conditions in the parametric design. Individual subject's expression of this activation pattern is quantified with the expression score and is obtained by mathematically projecting the covariance pattern in question onto a subject's task scan (an inner product) for both task levels. The expression score quantifies to what extent a subject expresses the activation pattern in a task scan with a single number for each task condition, and can then be used for further analysis. Even though the first OrT was performed in both age groups combined, the expression score quantifies the extent to which the network is activated in each participant, and was used to determine if each age group significantly expresses the network in analyses described in Section 2.9.

2.8.1.1. Permutation test. To ascertain whether an activation pattern exhibits a statistically significant ordinal trend, we conducted a permutation test. The task data were re-sampled and the condition assignment (single-task/task-switch) was 
broken, while leaving the subject assignment intact. The re-sampled data were submitted to our complete chain of analytic steps, and a covariance pattern was derived. The ordinal trend statistic (Habeck et al., 2005) which corresponds to the number of subjects who fail to show a monotonic increase from the single-task to the task-switch condition was computed for the covariance pattern. Executing these steps 1000 times generated a null-hypothesis histogram for the ordinal trend statistic. The p-level could be read off as the fraction of the iterations that produced a statistic smaller than our point estimate value.

2.8.1.2. Bootstrap resampling to assess stability of voxel loadings. To render an inferential judgment about the stability of the voxel loadings in the covariance pattern similar to a univariate map, we conducted another nonparametric bootstrap test. Contrary to the permutation test outlined before, the condition assignment was left intact and the data were re-sampled with replacement, i.e. some subjects were left out of the sample, while others were included more than once. This procedure approximates the natural variation incurred when sampling from the underlying distribution. The complete pattern-derivation recipe was applied to the re-sampled data. For each voxel, a $Z$-value was computed as

$Z=$ point estimate/STD

The point estimate was the voxel-loading for the covariance pattern obtained from the full sample, and the standard deviation was computed from the variability of the bootstrap results around this point estimate. We thresholded the resulting $Z$-map at $|Z|>1.96$, i.e. imposing a two-tailed p-level of $p<0.05$, and a cluster extent threshold of 100 voxels. Likely cytoarchitectonic labels for cluster maxima in these thresholded patterns were obtained using Talairach daemon software (Lancaster et al., 2000) and checked with visual inspection.

\subsubsection{Residualization}

The initial group-level covariance analysis identified a covariance pattern (or network) that was commonly expressed by both age groups. To investigate whether the two age groups activate additional networks distinct from the common network, data from each group was residualized with respect to the common network. The process of residualization subtracts the variance due to common network from the data, leaving only variability unaccounted for by the common network. If there is an additional network utilized for task performance, a significant ordinal trend should be identified in the residualized data. Thus, two separate covariance analyses were conducted on the two age groups' residualized data and the expression of any significant OrT network was correlated with behavioral variables. Any significant ordinal trend found in the residualized data in one group was also forward applied to the other group's residualized data to investigate whether the other group also expresses the same pattern.

\subsection{Relationships among age, pattern expression, and behavioral variables}

For any data that did not follow an ordinal trend as determined by the OrT permutation test, simple t-tests were performed to examine whether the mean expression was different from zero, which informed whether the group expressed the network at all. In separate ANOVA models, pattern expression for each identified pattern was treated as a dependent variable to examine the experimental effects of age group, condition, and their interactions.

The variables age, pattern expression, and behavioral measures were then examined with heterogeneous slopes general linear models (GLM) to test whether the relationship between pattern expression and behavioral variables, RT and accuracy, was different for the two age groups. The method of heterogeneous slopes (Kumar, Rakitin, Nambisan, Habeck, \& Stern, 2008; Siegel, 1956) was performed by starting with a general linear model that includes all of the possible interaction terms in addition to the main effects and then reducing the model by excluding all non-significant interactions. Thus, with RT and accuracy analyzed in separate models as the dependent variable, each of the full model comprised seven predictors: age group, condition, pattern expression, age group $\times$ condition, age group $\times$ pattern expression, condition $\times$ pattern expression, and age group $\times$ condition $\times$ pattern expression. Age difference in the relationship between pattern expression and behavioral performance would be supported by a significant interaction involving age and pattern expression. All tests were performed in Statistical Package for the Social Sciences (IBM SPSS Statistics 19.0)

\section{Results}

\subsection{Behavioral result}

Performance showed the expected Group and Condition effects. Percent accuracy was better in young $(M=95.63$, $S E=0.549)$ than older adults $(M=90.23, S E=1.14), F=6.72$, $p<0.05$, and also better in the single $(M=94.52, S E=0.910)$ than in the task-switch condition $(M=91.46, S E=0.919), F=34.15$, $p<0.01$. Reaction time also showed similar effects. Young adults $(M=874.1 \mathrm{~ms}, \quad S E=18.41)$ were faster than older adults $(M=1014.2 \mathrm{~ms}, S E=28.68), F=11.02, p<0.01$. Reaction time was faster in the single $(M=803.01 \mathrm{~ms}, S E=15.04)$ than in the task-switch condition $(M=1082.4 \mathrm{~ms}, S E=24.78), \quad F=184.8$, $p<0.01$. The interaction between Age and Condition, shown in Fig. 2, were marginally significant for both accuracy, $F=3.97$, $p=0.0523$, and RT, $F=3.46, p=0.0692$. The marginal interactions between Age and Condition are most likely due to the extensive training participants received prior to testing (Kramer et al., 1999).

Performance prior to and in the MRI scan (Phase) had an interactive effect with Condition. The decrease in accuracy from the single-task to the task-switch condition was more drastic pre(Single-task: $M=94.37, \quad S E=1.294 ; \quad$ Task-switch: $M=88.40$, $S E=1.590$ ) than in-scan (Single-task: $M=90.02, S E=2.31$; Taskswitch: $M=87.22, S E=2.144), F=11.42, p<0.01$. The overall RT was longer pre- $(M=1011.02 \mathrm{~ms}, S E=24.99)$ than in-scan $(M=874.37 \mathrm{~ms}, S E=22.84), F=55.78, p<0.01$, and the increase in RT from the single-task to the task-switch condition was slightly greater pre- (Single-task: $M=856.58 \mathrm{~ms}, S E=17.66$; Task-switch: $M=1165.46 \mathrm{~ms}, S E=34.92$ ) than in-scan (Singletask: $M=749.43 \mathrm{~ms}, \quad S E=21.97$; Task-switch: $M=999.32 \mathrm{~ms}$, $S E=31.23), F=13.87, p<0.01$.

Accuracy on Go and No-go trials also differed by Age Group, with a greater decrease in accuracy for Go trials in older adults (Young: No-go $M=99.3, S E=0.342$, Go $M=93.79, S E=1.57$; Old: No-go $M=98.5, S E=0.512$, Go $M=86.1, S E=3.35), F=6.2, p<0.05$.
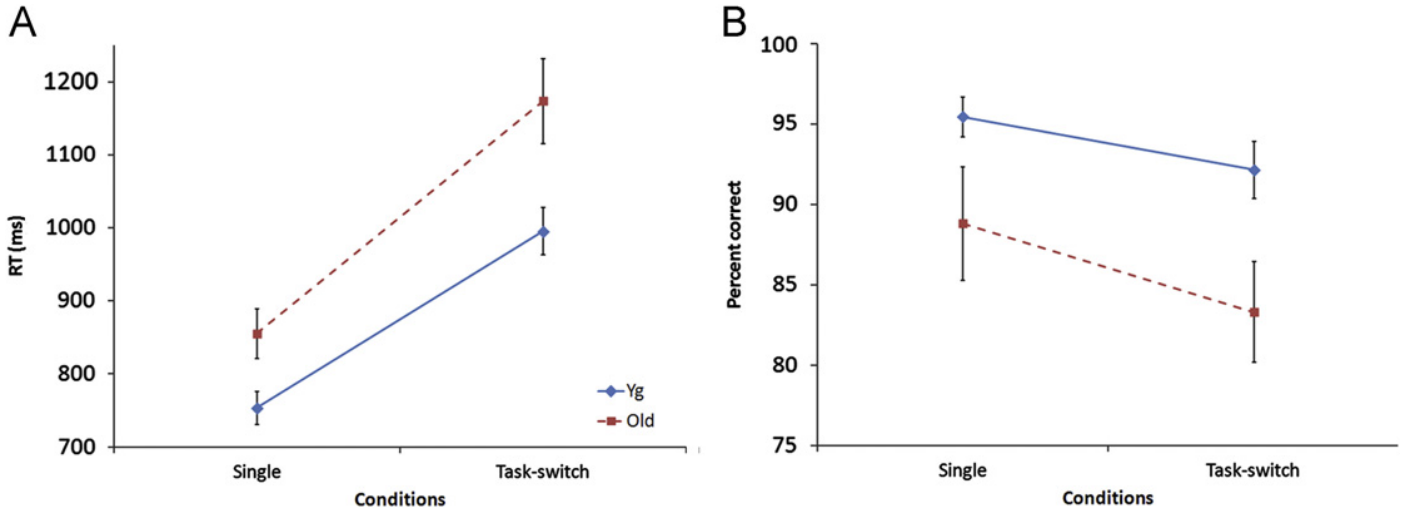

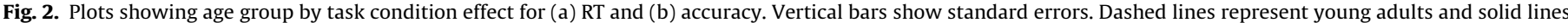
represent older adults. 


\section{2. fMRI OrT Patterns}

\subsubsection{Shared OrT pattern}

In data from both age groups, the OrT analysis identified a significant task-related pattern whose expression increased from the single-task to the task-switch condition, $p<0.001$ for 11 exceptions out of 47 participants that did not follow the ordinal trend. The positive weights in the pattern are regions that activate more from single-task to task-switch condition while the regions with negative weights show a reduction in activation from single-task to taskswitch condition. The regions with $z$ values above threshold in the positive weighted pattern include extensive bilateral cerebellum, the left middle occipital lobe extending into the precuneus, the right superior parietal lobule, the left prefrontal cortex (BA 9), the right superior occipital gyrus, and the right thalamus. See Table 2 and Fig. $3 \mathrm{~A}$ for more details. Negatively weighted regions include the right inferior parietal lobule extending into the superior temporal gyrus, the right precentral gyrus extending into the insula cortex, the right inferior frontal gyrus, bilateral superior temporal gyrus, the right SMA, the right anterior cingulate, the right medial frontal gyrus, the left posterior cingulate, the left putamen, and the left middle temporal gyrus.

With age group and condition as predictors, expression of the shared OrT pattern was higher in the task-switch condition $(M=0.320 \pm 0.044)$ than in the single-task condition $(M=0.221 \pm$ $0.033), F(1,45)=16.24, p<0.001$, but the age group effect was marginally significant, $F(1,45)=3.941, p=0.053$ (older adults: $M=0.343 \pm 0.052$; young adults: $M=0.198 \pm 0.051$ ), and the interaction between age group and condition was not significant, $F(1,45)=1.542, p=0.221$.

Univariate analysis of the Age by Condition effect was also performed but there were no areas where this interaction reached the threshold for significance. The OrT analysis was more sensitive than univariate approach because (1) it was not constrained by stringent thresholding to correct for voxel-wise multiple comparisons, and (2) it used a transformation that specifically maximizes the between condition differences in a monotonically increasing trend. These advantages are especially important when the between condition difference is small relative to the overall noise.

\subsubsection{Young-residual OrT pattern}

A significant OrT pattern was identified in the residualized young data ( $p<0.001$ for four exceptions out of 24 participants) in which the regions that increases activation with greater task difficulty (positively weighted regions) include two regions in the left middle frontal gyrus, bilateral anterior cingulate extending into the medial frontal gyrus, the left precentral gyrus, bilateral thalamus, the left inferior parietal lobule extending into the precuneus and the right cerebellum, and the regions that decreases activation with greater task difficulty (negatively weighted regions) include bilateral superior temporal gyrus, right posterior temporal gyrus extending into the occipital gyrus, bilateral inferior parietal lobule, the left occipital cortex, bilateral middle temporal gyrus, and the right occipital cortex extending into the cerebellum (see Table 3 and Fig. 3B for details).

A separate OrT pattern was not found in the residualized older adults' data ( $p=0.187$ for nine exceptions out of 23 participants). However, by forward applying the OrT pattern found in the residualized young adults' data to the residualized older adults' data, older adults do express the network in both task conditions (Single-task: $M=198.6 \pm 20.21, t=9.829, p<0.001$; Task-switch: $M=220.0 \pm 40.00, t=5.504, p<0.001)$ but their expression did not follow an ordinal trend (i.e. the expression of the pattern did not show a consistent trend with task difficulty) whereas the young adults' pattern expression did: age group by task condition interaction was significant: $F(1,45)=8.566, p<0.01$ (see Fig. $4 \mathrm{~A}$ for the mean values) with the Condition main effect also significant, $F(1,45)=15.63, p<0.001$ and the Age group main effect marginally significant, $F(1,45)=3.642, p=0.063$.

\subsection{Relationships among age, pattern expression, and behavioral variables}

Heterogeneous slopes test examined whether the relationship between pattern expression and the behavioral variables differed by age group and condition by first testing the predictors (Age group, Condition, and Network expression) and all of the interactions in a full-factorial model and then retesting the effects in the reduced model in which the non-significant interaction terms were excluded (Kumar et al., 2008). The test found

Table 2

Brain regions in the shared OrT network.

\begin{tabular}{|c|c|c|c|c|c|c|c|c|}
\hline Cluster \# & Region & Hem & BA & $x$ & $y$ & $z$ & $z$-value & $k$ \\
\hline \multicolumn{9}{|l|}{ Positive } \\
\hline 1 & Cerebellum & $\mathrm{L} / \mathrm{R}$ & NA & -20 & -74 & -26 & 2.96 & 4301 \\
\hline 2 & Mid occipital/Precuneus & $\mathrm{L}$ & $7 / 19$ & -30 & -78 & 36 & 2.87 & 251 \\
\hline 3 & Sup parietal lobule & $\mathrm{R}$ & 7 & 26 & -74 & 46 & 2.75 & 114 \\
\hline 4 & Prefrontal cortex & $\mathrm{L}$ & 9 & -38 & 20 & 40 & 2.60 & 129 \\
\hline 5 & Occipital gyrus & $\mathrm{R}$ & 19 & 34 & -78 & 28 & 2.56 & 133 \\
\hline 6 & Thalamus & $\mathrm{R}$ & NA & 2 & -14 & 12 & 2.35 & 51 \\
\hline \multicolumn{9}{|l|}{ Negative } \\
\hline 7 & Inf parietal lobule/Sup temporal gyrus & $\mathrm{R}$ & $40 / 42$ & 62 & -24 & 20 & 2.85 & 140 \\
\hline 8 & Precentral gyrus/ Insula cortex & $\mathrm{R}$ & $6 / 13$ & 48 & -8 & 10 & 2.76 & 542 \\
\hline 9 & Inf frontal gyrus & $\mathrm{R}$ & $45 / 47$ & 50 & 34 & 2 & 2.69 & 77 \\
\hline 10 & Sup temporal gyrus/Claustrum & $\mathrm{L}$ & 38 & -36 & -6 & -10 & 2.67 & 367 \\
\hline 11 & Sup temporal gyrus/hippocampus & $\mathrm{R}$ & 38 & 36 & 2 & -16 & 2.52 & 149 \\
\hline 12 & Supplementary motor area & $\mathrm{R}$ & 6 & 6 & -18 & 54 & 2.47 & 63 \\
\hline 13 & Anterior cingulate & $\mathrm{R}$ & 24 & 6 & 28 & 24 & 2.37 & 56 \\
\hline 14 & Medial frontal gyrus & $\mathrm{R}$ & 10 & 0 & 50 & 6 & 2.35 & 56 \\
\hline 15 & Posterior cingulate & $\mathrm{L}$ & 26 & -8 & -44 & 28 & 2.30 & 66 \\
\hline 16 & Putamen & $\mathrm{L}$ & NA & -24 & -4 & 10 & 2.25 & 79 \\
\hline 17 & Cingulate gyrus & $\mathrm{L} / \mathrm{R}$ & 24 & 4 & -8 & 42 & 2.16 & 61 \\
\hline 18 & Middle temporal gyrus & $\mathrm{L}$ & 37 & -42 & -64 & 0 & 2.14 & 53 \\
\hline
\end{tabular}

Note: Coordinates represent the local maxima in each cluster. $x, y, z=\mathrm{MNI}$ coordinates; Hem $=$ Hemisphere; $\mathrm{BA}=\mathrm{Brodmann}$ 's area $\mathrm{Inf}=$ inferior; Sup=superior; $k=$ cluster size; $\mathrm{NA}=$ not applicable; $\mathrm{L}=$ left; $\mathrm{R}=$ right. Cluster $\sharp$ s correspond to clusters in Fig. $3 \mathrm{~A}$. 


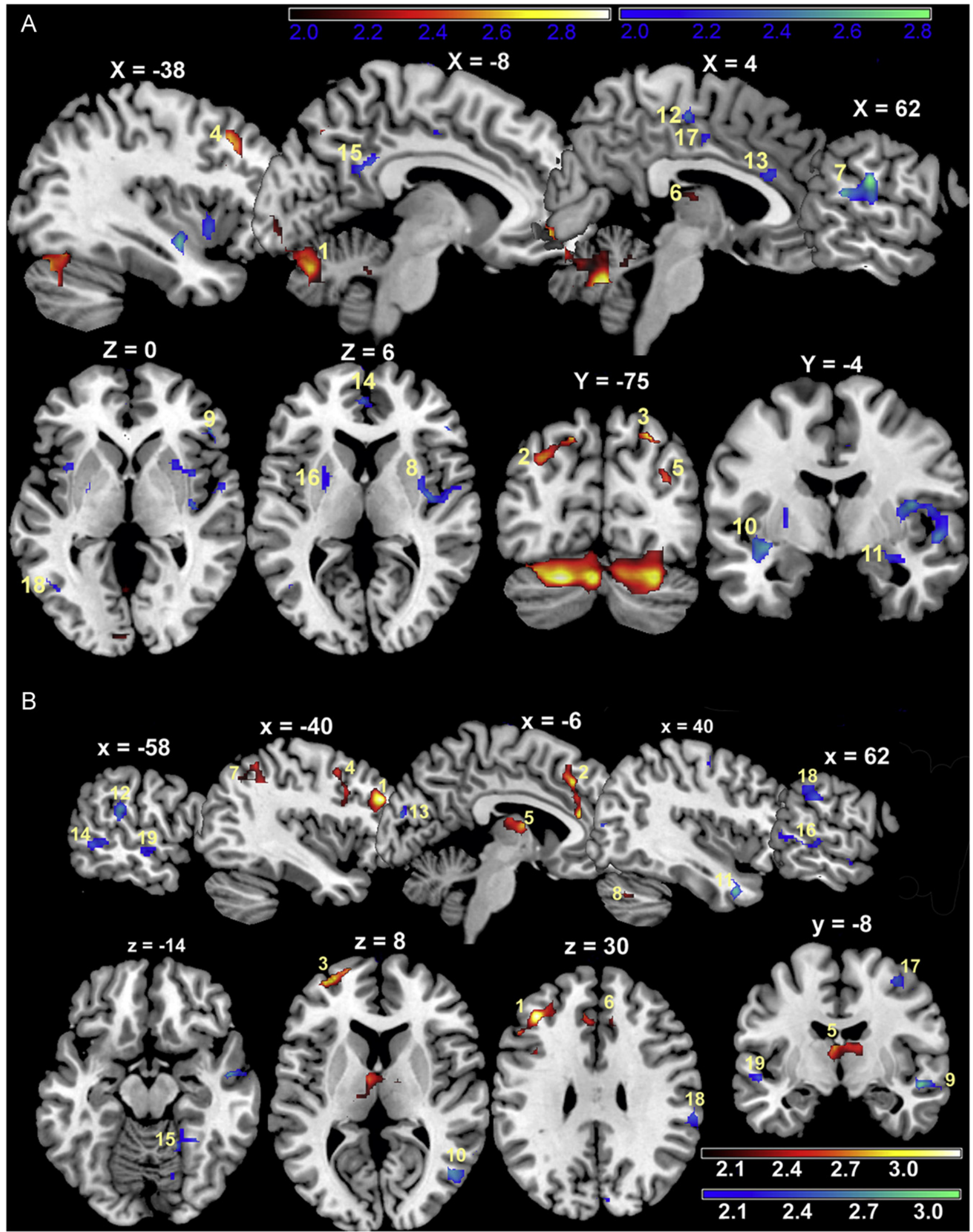

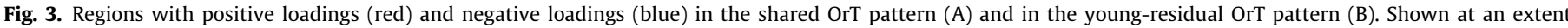

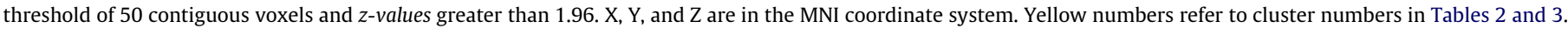

that the relationship between behavior and pattern expression was not different in the two age groups for both accuracy and RT, details are as follows. In the full model predicting accuracy, none of the predictors showed a significant effect while in the reduced model, in which all interactions were excluded, only age group showed a significant effect, $F(1,90)=10.83, p<0.01$. Similarly 
Table 3

Brain regions in the young-residual network.

\begin{tabular}{|c|c|c|c|c|c|c|c|c|}
\hline Cluster \# & Region & Hem & BA & $x$ & $y$ & $z$ & $z$-value & $k$ \\
\hline \multicolumn{9}{|l|}{ Positive } \\
\hline 1 & Middle frontal gyrus & $\mathrm{L}$ & $9 / 46$ & -38 & 34 & 30 & 3.32 & 288 \\
\hline 2 & Anterior cingulate/medial frontal gyrus & $\mathrm{L}$ & $24 / 32$ & -6 & 34 & 20 & 3.06 & 197 \\
\hline 3 & Middle frontal gyrus & $\mathrm{L}$ & 10 & -30 & 56 & 8 & 3.02 & 155 \\
\hline 4 & Precentral/middle frontal gyrus & $\mathrm{L}$ & $6 / 9$ & -38 & 10 & 36 & 3.00 & 76 \\
\hline 5 & Thalamus & $\mathrm{L}$ & NA & -4 & -6 & 12 & 2.95 & 375 \\
\hline 6 & Anterior cingulate/medial frontal gyrus & $\mathrm{R}$ & 32 & 10 & 28 & 38 & 2.79 & 120 \\
\hline 7 & Inf parietal lobule/precuneus & $\mathrm{L}$ & $7 / 40$ & -42 & -50 & 52 & 2.66 & 284 \\
\hline 8 & Cerebellum & $\mathrm{R}$ & - & 38 & -66 & -36 & 2.37 & 83 \\
\hline \multicolumn{9}{|l|}{ Negative } \\
\hline 9 & Sup temporal gyrus & $\mathrm{R}$ & 22 & 52 & -6 & -12 & 3.16 & 100 \\
\hline 10 & Posterior temporal/occipital gyrus & $\mathrm{R}$ & $19 / 37$ & 48 & -62 & 10 & 2.96 & 247 \\
\hline 11 & Sup temporal gyrus & $\mathrm{R}$ & 38 & 40 & 8 & -34 & 2.86 & 98 \\
\hline 12 & Inf parietal lobule & $\mathrm{L}$ & 40 & -58 & -32 & 22 & 2.70 & 105 \\
\hline 13 & Occipital cortex & $\mathrm{L}$ & $18 / 19$ & -6 & -88 & 18 & 2.60 & 101 \\
\hline 14 & Middle temporal gyrus & $\mathrm{L}$ & 21 & -60 & -52 & -2 & 2.54 & 80 \\
\hline 15 & Occipital cortex/cerebellum & $\mathrm{R}$ & $18 / \mathrm{NA}$ & 18 & -50 & -14 & 2.51 & 189 \\
\hline 16 & Middle temporal gyrus & $\mathrm{R}$ & 21 & 58 & -40 & -4 & 2.51 & 91 \\
\hline 17 & Precentral gyrus & $\mathrm{R}$ & $4 / 6$ & 36 & -8 & 56 & 2.46 & 69 \\
\hline 18 & Inf parietal lobule & $\mathrm{R}$ & 40 & 62 & -28 & 30 & 2.39 & 105 \\
\hline 19 & Sup temporal gyrus & $\mathrm{L}$ & 22 & -52 & -8 & -6 & 2.36 & 113 \\
\hline
\end{tabular}

Note: Coordinates represent the local maxima in each cluster. $x, y, z=$ MNI coordinates; Hem=Hemisphere; BA=Brodmann's area; Inf =inferior; Sup=superior; $k=$ cluster size; $\mathrm{NA}=$ not applicable; $\mathrm{L}=$ left; $\mathrm{R}=$ right. Cluster \#s correspond to clusters in Fig. $3 \mathrm{~B}$.
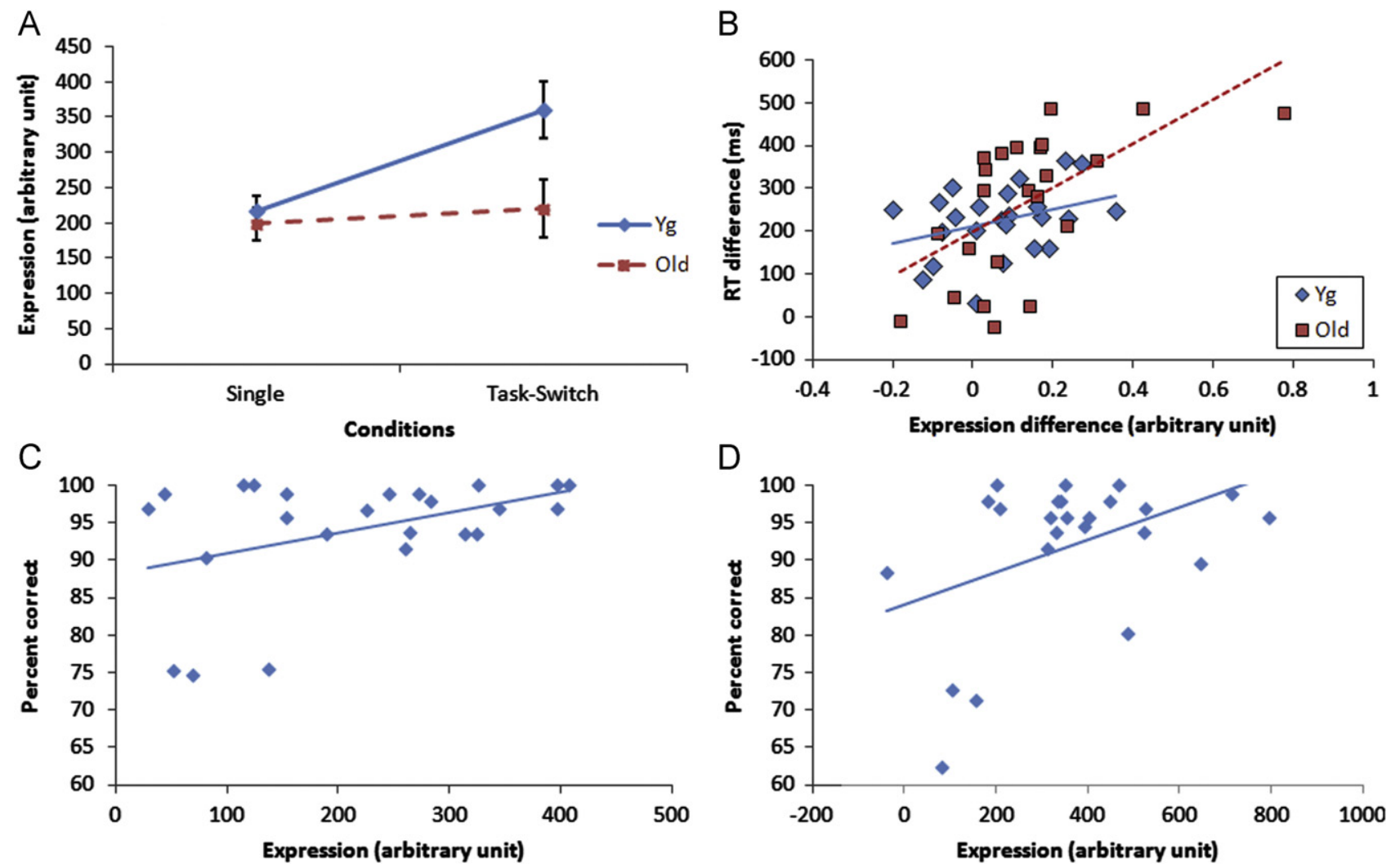

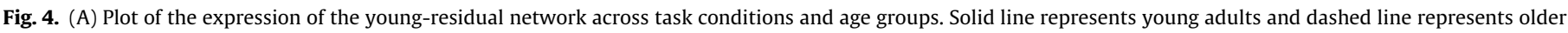

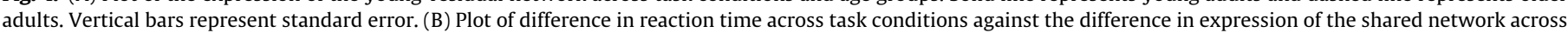

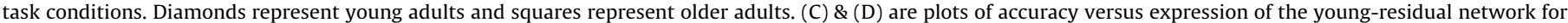
the single-task condition (C) and the task-switch condition (D).

for RT, none of the interaction terms in the full model was significant but the main effects condition, $F(1,86)=19.60, p<0.001$, and pattern expression, $F(1,86)=15.18, p<0.001$, were significant. In the reduced model for $\mathrm{RT}$, after excluding all interaction terms, all three main effects were significant (age group: $F(1,90)=11.54$, $p<0.01$; condition: $F(1,90)=47.00, p<0.001$; pattern expression: $F(1,90)=19.00, p<0.001)$. Thus, the network expression for the common pattern does not predict accuracy but it does predict RT with no age difference in the relationship between network expression and RT. 
To further explore the relationship between network expression and RT, differences between the two task conditions for both network expression and RT were examined with respect to age (i.e. in a ANOVA with age group and network expression difference as predictors of RT difference). In the full model, only the expression difference significantly predicted RT difference, $F(1,43)=12.32, p<0.01$, and in the reduced model with the age group by expression difference taken out of the model, expression difference was still the only significant predictor of RT difference, $F(1,44)=16.60, p<0.001$. Fig. 4B shows RT difference vs. expression difference across the two task levels. Correlation between the expression of the shared network and RT difference was $r(47)=0.537, p<0.001$. The difference in RT between task-switch and single-task blocks is not the same as the global switch cost, which has been defined as the difference between the RT in single-task blocks and non-switch RT in task-switch blocks. The correlation between RT difference and global switch cost in our study was highly significant, $r=0.837, p<0.001$ and global switch cost also significantly predicted expression difference. However, RT difference was used rather than global switch cost in our study because the measure is a more appropriate correlate of the neural patterns derived in our study in that our neural patterns included activity for each block in its entirety whereas global switch cost only consisted of portions of trials in each block.

For the young-unique network, difference of slopes tests showed that the expression of this network does not predict RT, in which the only significant predictor of RT in the full model was condition, $F(1,86)=8.648, p<0.01$, and in the reduced model, condition, $F(1,90)=42.58, p<0.001$, and age group, $F(1,90)=21.83, p<0.001$. The interactions involving age group and network expression were not significant in both models. Similar result was found for accuracy: no significant effect in the full model and only significant group effect in the reduced model, $F(1,90)=7.773, p<0.01$. However, the network expression does correlate with accuracy in young adults in both conditions (single-task: $r(24)=0.425, p<0.05$; taskswitch: $r(24)=0.430, p<0.05)$ as shown in Fig. $4 C$ and D.

\section{Discussion}

Our study examined whether there are similar as well as unique brain networks activated by young and older adults to perform task-switching and how the expression of these brain networks relate to behavioral performance in both age groups. Our data supports a shared task-related pattern strongly expressed by both young and older adults, in which not only the expression of the shared network correlated with reaction time in both age groups and in both conditions, differences in the expression of the shared pattern across the task conditions also correlated with the difference in RT between the single-task and the task-switching conditions in individual subjects. After residualizing out the shared network, an additional task-related pattern was first found in the residualized young adults' data the expression of which correlated with accuracy in both conditions only for young adults. In older adults, the young-residual network was expressed but their expression remained constant rather than appropriately increasing with greater task difficulty as shown in the young adults' data.

\subsection{Shared brain network in task-switching}

The positively weighted regions in the shared network consisted mostly of areas in the occipital cortex and the cerebellum, usually associated with basic visual and motor processes. As task difficulty increases, our data showed that activation in these basic input and output regions also increased. In addition, compared to young adults, elderly adults exhibited elevated activation levels in these basic processing regions. This is in contrast to the result reported by Cliff et al. (2012) in which flashing checkerboards of increasing presentation frequency were presented. Elderly adults showed decreased activation in the visual cortex with increasing frequency of stimulus presentation while activation in young adults increased with higher frequency. One potential source of the discrepancy is the passive viewing in Cliff et al. study versus the active task performance in our study. Actively performing a cognitive task seems to increase activation compared to young adults rather than lowered activation as was found during passive viewing. Our result further showed that greater activation increase in these regions was associated with longer RT increase from the single-task to the task-switching condition suggesting the existence of a relationship between RT duration and the intensity of activation in the basic processing regions.

The negatively weighted regions in the network decreased in activation with greater task difficulty. These included regions identified as part of the default mode network (Buckner, Andrews-Hanna, \& Schacter, 2008; Raichle et al., 2001): medial prefrontal cortex, including BA 10 and 24, hippocampus, and the inferior parietal lobule (BA 40). Activity in these brain areas have been found to correlate negatively with task-related activation (Shulman et al., 1997) and to be active when the brain was awake but at rest, a time when the brain engaged in self-related mental processes (Buckner \& Carroll, 2007; Spreng, Mar, \& Kim, 2009). Whether these regions deactivated as a function of default mode network the activation would need to be explored in separate connectivity analyses. In this case these regions deactivated as part of a network that included covarying areas of positive activation. Activation in other regions of the negatively weighted network, including the right inferior frontal gyrus, the supplementary motor area (SMA), and the putamen, may be negatively correlated with RT such that reduced activation in these regions is associated with longer RT, and conversely, greater activation was conducive to shorter RT. The right inferior frontal gyrus was reported to be involved in inhibitory processes while the SMA and the putamen participate in motor processes such as motor planning and motor sequence categorization (Seger, 2008).

Greater expression in elderly than young adults appears to suggest compensatory mechanism involved in task-switching, however, according to Stern et al. (2012) compensatory mechanisms should result in the use of an additional network that accompanies maintained performance. The elderly adults in our study performed slower and less accurate than young adults in our study, and except for the network shared with young adults no other network was identified in elderly adults. Reuter-Lorenz and Cappell's (2008) compensation-related utilization of neural circuits hypothesis (CRUNCH) model also hypothesized that greater activation should be associated with better performance in elderly adults, which was also not true in our study given that greater network expression was correlated with longer RT. Therefore, rather than a compensatory mechanism, greater expression in elderly adults is indicative of reduced efficiency in cognitive processing with older age. Whereas a certain level of activation is sufficient for young adults to process a task, elderly adults have to recruit the same processes to a greater extent.

Furthermore, the pattern expression-RT relationship is not different between the age groups, consistent with an agerelated degradation that follows a continuum with advancing age rather than aging as a pathological development. In other words, since expression of the primary network is predictive of RT throughout the two age groups with the same positive slope (increasing RT with increasing age), neuronal degradation appears to be a continuously deteriorating process extended from young adulthood to old age without any accelerated decline-a 
hypothesis that could be confirmed in a future longitudinal study. A steady rate of neural deterioration throughout lifetime may occur in a number of systems. White matter tract integrity is one potential candidate: Gold et al. (2010) found that fractional anisotropy, a measure of white matter integrity, in the frontoparietal regions mediated RT in task-switching. Aging research in non-human primates identified greater degeneration in myelin sheaths that also correlates with cognitive decline (Peters \& Kemper, 2011).

\subsection{Young-residual network}

While age-related degradation in activating the shared network follows a continuum throughout the aging process, our data shows that functioning of the young-residual network is disrupted in older adults, which may have contributed to the performance decline in older adults. Our results show that greater increase in activation across conditions of the young-residual network is associated with better accuracy in both task conditions only for young adults. Even though older adults did activate the young-residual network their expression of the network remains low and did not increase with greater task difficulty. Elders' accuracy was worse than young adults' and did not correlate with expression of the young-residual network. The inability to increase network expression with task difficulty may have contributed to their relatively poorer performance. Contrary to the shared network, the disruption of proper functioning in the young-residual network may constitute a pathological change associated with age, and may be due to damages in the structural integrity of the aged brain preventing appropriate activation of the network.

The regions within the young-residual network were reported by Braver, Reynolds, and Donaldson (2003) to be associated with task-switching. The left SMA (BA 6), the left superior parietal (BA 7 ), and the left dorsolateral PFC (BA 9) were associated with transient activation while the anterior cingulate (BA 24) was associated with sustained activation. Jimura and Braver (2010) found that older participants have lower sustained activation in the anterior prefrontal cortex (BA 10/46) than young participants. Their result is consistent with the greater expression of the network in young than older adults in our study since our analysis was based on blocked design which captures sustained activation. Our study extends previous findings by showing that even though older adults have lower activation in the anterior prefrontal cortex throughout task-switching, they do not increase the level of activation with task difficulty as young adults do and their level of accuracy no longer correlates with the activation of the region.

\subsection{Age-related changes in brain activity}

Overall, older participants showed increased activity with higher task demand in the shared network, a network that consisted mostly of posterior activations such as in the occipital lobe and the cerebellum, while expressing disruptively low activity in the youngresidual network, which consisted mostly of anterior activation such as the prefrontal areas and the precentral gyrus. The reduced expression of a pattern consisting of prefrontal activation and the increased expression of a posterior network was consistent with the hypothesis that age-related deficit in task-switching was due to disruption of prefrontally mediated processes such as response selection (Meiran \& Gotler, 2001; Meiran, Gotler, \& Perlman, 2001). Through a series of experiments manipulating Cue-Target interval and Response-Cue interval as well as quantitative modeling, Meiran et al. (2001) provided evidence that aging had the largest effect on the residual cost of task-switching, characterized by increased RT during task-switching even when given sufficient preparation time (long
Cue-Target interval). By quantitative modeling (Meiran \& Gotler, 2001) and confirmed with measuring the effect of response compatibility on residual cost, the authors suggested that residual cost was partly attributable to response selection, a process that had been reported to be mediated by prefrontal regions (Schumacher, Cole, \& D'Esposito, 2007). Thus the authors concluded that aging decline in task-switching is mainly due to a deficit in response selection processing. This conclusion is also consistent with the disruption of an accuracy-related activation pattern in our elderly group. Response selection processing directly contributes to the accuracy of responses, the disruption of which lowers accuracy in task performance, as was found in our result.

A potential contributing cause of lowered frontal-pattern expression may be that greater age-related changes in white matter health have been found in the frontal regions (Madden, Bennett, \& Song, 2009; Pfefferbaum, Adalsteinsson, \& Sullivan, 2005). Older adults have lower white matter integrity in the anterior regions than young adults but minimal age effect in the posterior regions (Pfefferbaum et al., 2005), suggesting a deterioration of white matter health in the anterior regions and more preserved white matter structure in the posterior regions. Disruption of network activation in older adults as shown in their expression of the young-residual network may be attributed to breakdown of the myelin sheath which can disrupt the timing of signals and proper integration of signals in task-related processes (Salat, 2011). Given the important role white matter health may have in cognitive processing, future studies should incorporate measures of white matter health in predicting the relationship between age-related cognitive decline and functional brain activation.

\section{Conclusion}

Our study provides one of the first multivariate results that identifies a task-switching network shared by young and older adults, the difference across task levels in the expression of which correlates with RT and RT difference across single-task and taskswitching conditions, and an additional network with disrupted activation in older adults but the expression of which correlates with accuracy in young adults. The results suggest that different brain networks follow distinct age-related trajectories, ranging from healthy maintenance to complete disruption of a network with increasing age.

\section{Acknowledgments}

This work was supported NIA R01AG026158, NIA T32 AG00261, and UL1 RR024156. We thank Aaron Reuben and Amanda Phingbodhipakkiya for their help in data collection and Aleksey Orekhov for his help in fMRI data processing.

\section{References}

Allport, A., Styles, E. A., \& Hsieh, S. (1994). Shifting intentional set: Exploring the dynamic control of tasks. In: C. Umilta, \& M. Moscovitch (Eds.), Attention and performance XV: Conscious and nonconscious information processing (pp. 421-452). Cambridge, MA: MIT Press

Braver, T. S., Reynolds, J. R., \& Donaldson, D. I. (2003). Neural mechanisms of transient and sustained cognitive control during task switching. Neuron, 39(4), 713-726.

Buckner, R. L., Andrews-Hanna, J. R., \& Schacter, D. L. (2008). The brain's default network: anatomy, function, and relevance to disease. Annals of the New York Academy of Sciences, 1124, 1-38.

Buckner, R. L., \& Carroll, D. C. (2007). Self-projection and the brain. Trends in Cognitive Sciences, 11(2), 49-57.

Cliff, M., Joyce, D. W., Lamar, M., Dannhauser, T., Tracy, D. K., \& Shergill, S. S. (2012) Aging effects on functional auditory and visual processing using fMRI with 
variable sensory loading. Cortex: A Journal Devoted to the Study of the Nervous System and Behavior, http://dx.doi.org/10.1016/j.cortex.2012.04.003.

Crone, E. A., Wendelken, C., Donohue, S. E., \& Bunge, S. A. (2006). Neural evidence for dissociable components of task-switching. Cerebral Cortex, 16(4), 475-486.

Drag, L. L., \& Bieliauskas, L. A. (2009). Contemporary review 2009: Cognitive aging. Journal of Geriatric Psychiatry and Neurology, 23(2), 75-93.

Eyler, L. T., Sherzai, A., Kaup, A. R., \& Jeste, D. V. (2011). A review of functional brain imaging correlates of successful cognitive aging. Biological Psychiatry, 70(2), 115-122.

Friston, K. J. (2007). Statistical parametric mapping: The analysis of functional brain images. London: Academic.

Gold, B. T., Powell, D. K., Xuan, L., Jicha, G. A., \& Smith, C. D. (2010). Age-related slowing of task switching is associated with decreased integrity of frontoparietal white matter. Neurobiology of Aging, 31(3), 512-522.

Habeck, C., Rakitin, B. C., Moeller, J., Scarmeas, N., Zarahn, E., Brown, T., et al. (2005). An event-related fMRI study of the neural networks underlying the encoding, maintenance, and retrieval phase in a delayed-match-to-sample task. Cognitive Brain Research, 23(2-3), 207-220.

Jimura, K., \& Braver, T. S. (2010). Age-related shifts in brain activity dynamics during task switching. Cerebral Cortex, 20(6), 1420-1431.

Karayanidis, F., Whitson, L. R., Heathcote, A., \& Michie, P. T. (2011). Variability in proactive and reactive cognitive control processes across the adult lifespan. Frontiers in Psychology, 2, 318.

Koechlin, E., Ody, C., \& Kouneiher, F. (2003). The architecture of cognitive control in the human prefrontal cortex. Science (New York, NY), 302(5648), 1181-1185.

Kramer, A. F., Hahn, S., \& Gopher, D. (1999). Task coordination and aging: explorations of executive control processes in the task switching paradigm. Acta Psychologica, 101(2-3), 339-378.

Kray, J., \& Lindenberger, U. (2000). Adult age differences in task switching. Psychology and Aging, 15(1), 126-147.

Kumar, A., Rakitin, B. C., Nambisan, R., Habeck, C., \& Stern, Y. (2008). The responsesignal method reveals age-related changes in object working memory. Psychology and Aging, 23(2), 315-329.

Lancaster, J. L., Woldorff, M. G., Parsons, L. M., Liotti, M., Freitas, C. S., Rainey, L., et al. (2000). Automated Talairach atlas labels for functional brain mapping. Human Brain Mapping, 10(3), 120-131.

Lustig, C., Shah, P., Seidler, R., \& Reuter-Lorenz, P. A. (2009). Aging, training, and the brain: A review and future directions. Neuropsychological Review, 19(4), 504-522.

Macwhinney, B., Cohen, J., \& Provost, J. (1997). The PsyScope experiment-building system. Spatial Vision, 11(1), 99-101.

Madden, D. J., Bennett, I. J., \& Song, A. W. (2009). Cerebral white matter integrity and cognitive aging: Contributions from diffusion tensor imaging. Neuropsychological Review, 19(4), 415-435.

Madden, D. J., Costello, M. C., Dennis, N. A., Davis, S. W., Shepler, A. M., Spaniol, J., et al. (2010). Adult age differences in functional connectivity during executive control. NeuroImage, 52(2), 643-657.

Mayr, U. (2001). Age differences in the selection of mental sets: The role of inhibition, stimulus ambiguity, and response-set overlap. Psychology and Aging, 16(1), 96-109.

Mayr, U., \& Keele, S. W. (2000). Changing internal constraints on action: the role of backward inhibition. Journal of Experimental Psychology: General, 129(1), 4-26.

McIntosh, A. R., Bookstein, F. L., Haxby, J. V., \& Grady, C. L. (1996). Spatial pattern analysis of functional brain images using partial least squares. NeuroImage 3(3 Pt 1), 143-157.
Meiran, N. (2000). Reconfiguration of stimulus task sets and response tasks sets during task-switching. In: S. Monsell, \& J. Driver (Eds.), Attention and performance XVIII: Control of cognitive performance. Cambridge, MA: MIT Press.

Meiran, N., \& Gotler, A. (2001). Modelling cognitive control in task switching and ageing. European Journal of Cognitive Psychology, 13, 165-186.

Meiran, N., Gotler, A., \& Perlman, A. (2001). Old age is associated with a pattern of relatively intact and relatively impaired task-set switching abilities. The Journal of Gerontology. Series B, Psychological Sciences and Social Sciences, 56(2), P88-P102.

Peters, A., \& Kemper, T. (2011). A review of the structural alterations in the cerebral hemispheres of the aging rhesus monkey. Neurobiology of Aging, http://dx.doi.org/10.1016/j.neurobiolaging.2011.11.015

Pfefferbaum, A., Adalsteinsson, E., \& Sullivan, E. V. (2005). Frontal circuitry degradation marks healthy adult aging: Evidence from diffusion tensor imaging. NeuroImage, 26(3), 891-899.

Raichle, M. E., MacLeod, A. M., Snyder, A. Z., Powers, W. J. Gusnard, D. A., \& Shulman, G. L. (2001). A default mode of brain function. Proceedings of the National Academy of Sciences of the United States of America, 98(2), 676-682.

Rajah, M. N., \& D'Esposito, M. (2005). Region-specific changes in prefronta function with age: A review of PET and fMRI studies on working and episodic memory. Brain, 128(Pt 9), 1964-1983.

Rakitin, B., \& Stern, Y. The effects of contextual, episodic, response inhibition and response-selection executive demands on choice decisions in the young and elderly.

Reuter-Lorenz, P. A., \& Cappell, K. A. (2008). Neurocognitive aging and the compensation hypothesis. Current Directions in Psychological Science, 17(3), 177-182.

Rogers, R. D., \& Monsell, S. (1995). Costs of a predictable switch between simple cognitive tasks. Journal of Experimental Psychology: General, 124(2), 207-231.

Salat, D. H. (2011). The declining infrastructure of the aging brain. Brain Connectivity, 1(4), 279-293.

Salthouse, T. A. (1996). The processing-speed theory of adult age differences in cognition. Psychological Review, 103(3), 403-428.

Schumacher, E. H., Cole, M. W., \& D'Esposito, M. (2007). Selection and maintenance of stimulus-response rules during preparation and performance of a spatial choice-reaction task. Brain Research, 1136(1), 77-87.

Seger, C. A. (2008). How do the basal ganglia contribute to categorization? Their roles in generalization, response selection, and learning via feedback. Neuroscience and Biobehavioral Reviews, 32(2), 265-278.

Shulman, G. L., Fiez, J. A., Corbetta, M., Buckner, R. L., Miezin, F. M., Raichle, M. E., et al. (1997). Common blood flow changes across visual tasks: II. Decreases in cerebral cortex. Journal of Cognitive Neuroscience, 9, 648-663.

Siegel, S. (1956). Nonparametric statistics for the behavioral sciences. New York: McGraw-Hill.

Spreng, R. N., Mar, R. A., \& Kim, A. S. (2009). The common neural basis of autobiographical memory, prospection, navigation, theory of mind, and the default mode: A quantitative meta-analysis. Journal of Cognitive Neuroscience, 21(3), 489-510.

Stern, Y., Rakitin, B. C., Habeck, C., Gazes, Y., Steffener, J., Kumar, A., et al. (2012). Task difficulty modulates young-old differences in network expression. Brain Research, http://dx.doi.org/10.1016/j.brainres.2011.11.061.

Verhaeghen, P., \& Cerella, J. (2002). Aging, executive control, and attention: A review of meta-analyses. Neuroscience and Biobehavioral Reviews, 26(7) 849-857.

Worsley, K. J., Poline, J. B., Friston, K. J., \& Evans, A. C. (1997). Characterizing the response of PET and fMRI data using multivariate linear models. Neurolmage, 6(4), 305-319. 